\title{
Definición diagnóstica en una familia con malattia leventinese en Colombia
}

Nancy Gelvez', Paula Hurtado-Villa², Silvia Flórez ${ }^{3}$, Anne Charlotte Brieke ${ }^{4}$, Francisco Rodríguez ${ }^{4}$, Ana María Bertolotto ${ }^{5,6}$, Martha L. Tamayo ${ }^{1}$

${ }^{1}$ Instituto de Genética Humana, Pontificia Universidad Javeriana, Bogotá D.C., Colombia

${ }^{2}$ Facultad de Ciencias de la Salud, Pontificia Universidad Javeriana, Cali, Colombia

${ }^{3}$ Independiente, Cúcuta, Colombia

${ }^{4}$ Fundación Oftalmológica Nacional, Escuela de Medicina y Ciencias de la Salud, Universidad del Rosario, Bogotá, D.C., Colombia

${ }^{5}$ Departamento de Pediatría, Facultad de Medicina, Pontificia Universidad Javeriana, Bogotá, D.C., Colombia

${ }^{6}$ Servicio de Pediatría, Hospital Universitario San Ignacio, Bogotá, D.C., Colombia

La malattia leventinese es una enfermedad hereditaria autosómica dominante, cuyos síntomas se inician entre la segunda y la cuarta décadas de la vida. Se caracteriza por la aparición de drusas localizadas entre el epitelio pigmentario de la retina y la membrana de Bruch; suele reducir la visión drásticamente y progresar a ceguera. La variante patogénica p.Arg345Trp en el gen EFEMP1 se ha asociado con esta enfermedad.

Se presenta aquí la caracterización clínica y molecular de una familia con malattia leventinese mediante un manejo integral que involucró a oftalmólogos, pediatras y genetistas, lo que es de gran importancia, ya que el fenotipo de esta enfermedad suele confundirse con la degeneración macular. A todos los individuos de la familia se les hizo la evaluación oftalmológica con imágenes diagnósticas de retina y extracción de ADN a partir de una muestra de sangre periférica. Todos los exones del gen EFEMP1 se amplificaron y secuenciaron. La variante patogénica p.Arg345Trp se identificó en los individuos afectados. Este es el primer reporte de malattia leventinese en una familia con la variante patogénica p.Arg345Trp en Colombia. El diagnóstico molecular de las distrofias retinianas es fundamental para diferenciar este tipo de enfermedades.

Recibido: 06/07/2020

Publicado: 08/03/202

Citación:

Gelvez N, Hurtado-Villa P, Flórez S, Brieke AC Rodríguez F, Bertolotto AM, Tamayo ML. Definición diagnóstica en una familia con malattia leventinese en Colombia. Biomédica. 2021;41:388-95. https://doi.org/10.7705/biomedica.5604

\section{Correspondencia:}

Marta L. Tamayo, Pontificia Universidad Javeriana, Carrera 7 No 40-62, edificio 32, Bogotá, D.C. Colombia

Teléfono: (571) 320 8320, extensión 2793

mtamayo@javeriana.edu.co

\section{Contribución de los autores:}

Nancy Gelvez: estudios moleculares y correlación genotipo-fenotipo

Paula Hurtado-Villa: estudios moleculares, correlación genotipo-fenotipo y asesoría genética Silvia Flórez y Francisco Rodríguez: diagnóstico clínico

Anne Charlotte Brieke: diagnóstico clínico, recopilación de exámenes paraclínicos Ana María Bertolotto: correlación genotipo-fenotipo y recopilación de exámenes paraclínicos

Martha L. Tamayo: correlación genotipo-fenotipo y asesoría genética

Financiación:

Este proyecto fue financiado por el Ministerio de Ciencia, Tecnología e Innovación, proyecto 120372453645, contrato 656-2015 "Programa de atención integral a familias con enfermedades huérfanas con componente visual y auditivo - AIVA" y la Fundación Derecho a la Desventaja (FUNDALDE), Bogotá, D.C., Colombia.

Conflicto de intereses:

Los autores manifiestan no tener conflicto de intereses.
Palabras clave: distrofias retinianas; epitelio pigmentario de la retina; degeneración macular; retina.

\section{Diagnostic definition of malattia leventinese in a family from Colombia}

The malattia leventinese is an autosomal dominant inherited disease whose symptoms appear between the second and fourth decades of life. It is characterized by the appearance of drusen located between the retinal pigment epithelium and the Bruch membrane. It is usually associated with low vision and may progress to blindness. The pathogenic variant p.Arg345Trp in the EFEMP1 gene has been associated with this disease. We characterized clinically and molecularly a family with malattia leventinese using a comprehensive approach that involved ophthalmologists, pediatricians, and geneticists. This approach is of great importance since the phenotype of this disease is often confused with macular degeneration. All family members underwent ophthalmological evaluation and DNA extraction from a peripheral blood sample. All exons of the EFEMP1 gene were amplified and sequenced. The pathogenic variant p.Arg345Trp was identified in affected individuals in this family.

This is the first report of malattia leventinese in a family with the p.Arg345Trp pathogenic variant in Colombia. The molecular diagnosis of retinal dystrophies is essential to differentiate this type of pathology.

Keywords: Retinal dystrophies; retinal pigment epithelium; macular degeneration; retina.

La malattia leventinese es una rara enfermedad degenerativa de herencia autosómica dominante que afecta la mácula (1-3) y causa pérdida grave de la visión o ceguera legal (4). También se la conoce como distrofia retiniana en panal de abejas de Doyne.

La enfermedad generalmente se manifiesta entre los 20 y los 40 años de edad, pero puede aparecer en la niñez o en la tercera edad (2). Un rasgo característico temprano de la malattia leventinese es la presencia en distribución radial de unos depósitos extracelulares denominados drusas, que suelen tener aspecto de "panal de abejas" y que se acumulan entre el 
epitelio pigmentario de la retina y la membrana de Bruch $(1,2)$. En una etapa más tardía de la enfermedad, se presenta una variedad de características clínicas e histopatológicas, incluidos la atrofia geográfica, los cambios en la pigmentación y la nueva irrigación coroidea, los cuales pueden llevar a disminución de la agudeza visual (2).

La malattia leventinese fue descrita por primera vez en individuos originarios del valle Leventina, en el sur de Suiza, de ahí su nombre (5). Hasta el momento, solo se ha reportado una variante patogénica en el gen EFEMP1 como causante de la enfermedad, la c.1033C>T/p.Arg345Trp $(1,6,7)$, la cual se ha detectado en población sueca, británica, australiana, norteamericana, japonesa y coreana (8).

Las drusas típicas de esta condición son acumulaciones de material extracelular, localzadas inmediatamente debajo del epitelio pigmentario de la retina (9), las cuales pueden ser una manifestación del proceso normal de envejecimiento o representar un signo importante asociado con enfermedades de la retina (10). La degeneración macular relacionada con la edad se caracteriza actualmente por la presencia de drusas y muchos fenotipos de drusas retinianas se agrupan bajo esta denominación. Esta es una enfermedad heterogénea y se han identificado hasta 34 loci diferentes (11). Son pocos los reportes relacionados con la malattia leventinense, lo que hace interesante su estudio y diferenciación diagnóstica con respecto a la degeneración macular relacionada con la edad.

En el presente estudio, se hicieron la evaluación oftalmológica completa y el estudio molecular del gen EFEMP1 en una familia colombiana con tres individuos afectados.

\section{Población de estudio}

Se incluyeron cinco individuos: tres mujeres afectadas y dos individuos sanos. Esta familia es natural de Barranquilla y en la consulta de genética médica se logró determinar que tenía ancestros del sur de Suiza (figura 1).

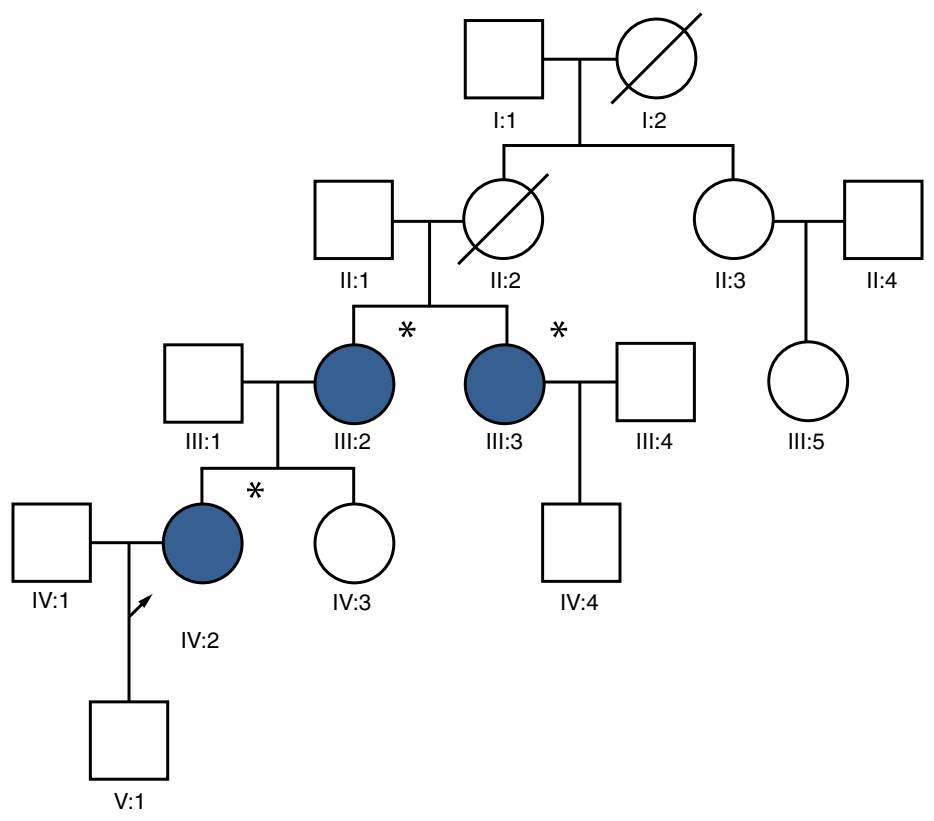

Figura1. Árbol genealógico de una familia colombiana con malattia leventinese 


\section{Evaluación clínica}

Todos los individuos fueron evaluados por un oftalmólogo especializado en retina y vítreo. Se les practicaron exámenes paraclinicos a dos de las tres mujeres afectadas (figura 1), pero en la tercera no fue posible hacerlo. En el caso de la paciente IV:2, estos se hicieron a los 27 años de edad y, en la paciente III:2, a los 41 años. Los exámenes incluyeron campo visual, angiografía con fluoresceína y electrorretinograma. El análisis molecular se realizó en los laboratorios del Instituto de Genética Humana de la Pontificia Universidad Javeriana Bogotá.

\section{Análisis del gen EFEMP1}

Se tomaron muestras de sangre periférica a los cinco individuos incluidos en el estudio. El ADN se extrajo mediante la técnica de fenol-cloroformo y se hicieron análisis de secuenciación bidireccional de Sanger de los 12 exones del gen EFEMP1.

Las condiciones de temperatura fueron las siguientes: $95^{\circ} \mathrm{C}$ durante 10 segundos, $50^{\circ} \mathrm{C}$ durante 5 segundos y $60^{\circ} \mathrm{C}$ durante 4 minutos para 35 ciclos. La precipitación de la reacción de secuenciación se hizo con el método de etanol. Las secuencias se analizaron con un equipo ABI-PRISM 3100-Avant ${ }^{\mathrm{TM}}$ (Applied Biosystems) usando el estuche de secuenciación BigDye Terminator ${ }^{\mathrm{TM}}$, versión 3.1 (Applied Biosystems). Por último, se analizaron las secuencias con el programa SeqScape ${ }^{\mathrm{TM}}$ (Applied Biosystems). El procedimiento utilizado para la secuenciación fue el siguiente: una vez obtenido el producto de PCR, se purificó y se hizo la reacción de secuenciación con $2 \mu \mathrm{l}$ de BigDye, $2 \mu \mathrm{l}$ de solución tampón $5 \mathrm{X}, 1 \mu \mathrm{l}$ de iniciador y $5 \mu \mathrm{l}$ del producto purificado.

La secuencia consenso utilizada para el gen EFEMP1 fue la ENST00000355426.8, obtenida en https://www.ensembl.org/Homo_ sapiens/Transcript/Exons?db=core;g=ENSG00000115380;r=2:55865967$55924139 ; \mathrm{t}=$ ENST00000355426.

\section{Consideraciones éticas}

Todos los individuos incluidos firmaron el consentimiento informado, aceptando la toma de muestras y la utilización de los datos de la historia clínica, así como su publicación. El estudio fue aprobado por el Comité de Ética de la Pontificia Universidad Javeriana. El protocolo se apega a la normatividad vigente contemplada en la Declaración de Helsinki de Fortaleza (Brasil) 2013 y la Resolución 8430 de 1993 del Ministerio de Salud de Colombia.

\section{Hallazgos clínicos}

Los exámenes paraclínicos se hicieron en dos de las tres mujeres afectadas de la familia (figura 1, cuadro 1). La paciente IV:2 fue valorada por presentar nictalopía como primer síntoma; refirió que su madre y una tía presentaban la enfermedad macular (III:2 y III:3). La mujer tenía alteración bilateral del epitelio pigmentario de la retina, con pigmentación fina, adelgazamiento retiniano macular, visualización de los vasos coroideos y drusas alrededor de la mácula (figura 2). Las alteraciones detectadas eran sugestivas de tres diagnósticos: a) drusas laminares hereditarias, b) retinitis punctata albecens, una variante de la retinosis pigmentosa, o c) retina pecosa (grupo de enfermedades caracterizado por múltiples 
lesiones blancoamarillentas en el fondo de ojo (fundus albipunctatus, fundus flavimaculatus, drusas familiares y retina en "flecos" de Kandori). El seguimiento del caso evidenció que la enfermedad progresaba y se asociaba clínicamente con disminución de la visión.

La paciente III:2 presentaba disminución progresiva de la visión desde los 34 años. El diagnóstico inicial fue de fundus flavimaculatus, ya que en la angiografía que le practicaron a esa edad se reportó hiperfluorescencia del área macular con acumulaciones pigmentarias localizadas, que parecían corresponder a una degeneración macular relacionada con la edad y probablemente asociada con un fundus albipuntatus. Posteriormente, a la edad de 41 años, recibió un segundo diagnóstico posible, el de distrofia de retina (enfermedad de Stargardt) (cuadro 1) y, a los 48 años, en otra angiografía con fluoresceína, se evidenció una hiperfluorescencia moteada en toda el área macular que permaneció estable a lo largo de las fases de estudio, así como una hiperfluorescencia relativa del nervio óptico en ambos ojos.

Cuadro 1. Resultados de los exámenes clínicos y paraclínicos de los individuos afectados

\begin{tabular}{|c|c|c|}
\hline & Paciente IV:2 & Paciente III:2 \\
\hline Edad (años) & 27 & 41 \\
\hline Agudeza visual & OD: $20 / 25 ;$ OI: $20 / 25$ & OD: $20 / 60 ;$ OI: $20 / 25$ \\
\hline Electrorretinograma & $\begin{array}{l}\text { Estimulación fotópica y escotópica } \\
\text { normal en ambos ojos }\end{array}$ & $\begin{array}{l}\text { Estimulación fotópica y escotópica } \\
\text { disminuida en ambos ojos }\end{array}$ \\
\hline $\begin{array}{l}\text { Angiografía con } \\
\text { fluoresceína }\end{array}$ & $\begin{array}{l}\text { Múltiples puntos amarillentos } \\
\text { hiperfluorescentes en patrón } \\
\text { radiado sugestivos de drusas en } \\
\text { polo posterior en ambos ojos }\end{array}$ & $\begin{array}{l}\text { Región hiperfluorescente en el área } \\
\text { de la fóvea que alterna con puntos } \\
\text { hiperfluorescentes alrededor en } \\
\text { ambos ojos }\end{array}$ \\
\hline \multirow[t]{2}{*}{ Campimetría } & $\begin{array}{l}\text { Defecto peripapilar asociado con } \\
\text { defecto macular incipiente en } \\
\text { ambos ojos }\end{array}$ & $\begin{array}{l}\text { OD: prueba de escasa confiabilidad } \\
\text { por numerosas pérdidas de fijación. } \\
\text { Los cambios sugieren disminución } \\
\text { generalizada de la sensibilidad } \\
\text { asociada con defecto temporal } \\
\text { superior profundo. }\end{array}$ \\
\hline & & $\begin{array}{l}\text { Ol: escasa confiabilidad por } \\
\text { numerosas pérdidas de fijación. } \\
\text { Los cambios sugieren disminución } \\
\text { generalizada de la sensibilidad } \\
\text { asociada con defecto temporal } \\
\text { superior profundo y defecto } \\
\text { paracentral inferior moderado. }\end{array}$ \\
\hline
\end{tabular}

OD: ojo derecho; OI: ojo izquierdo

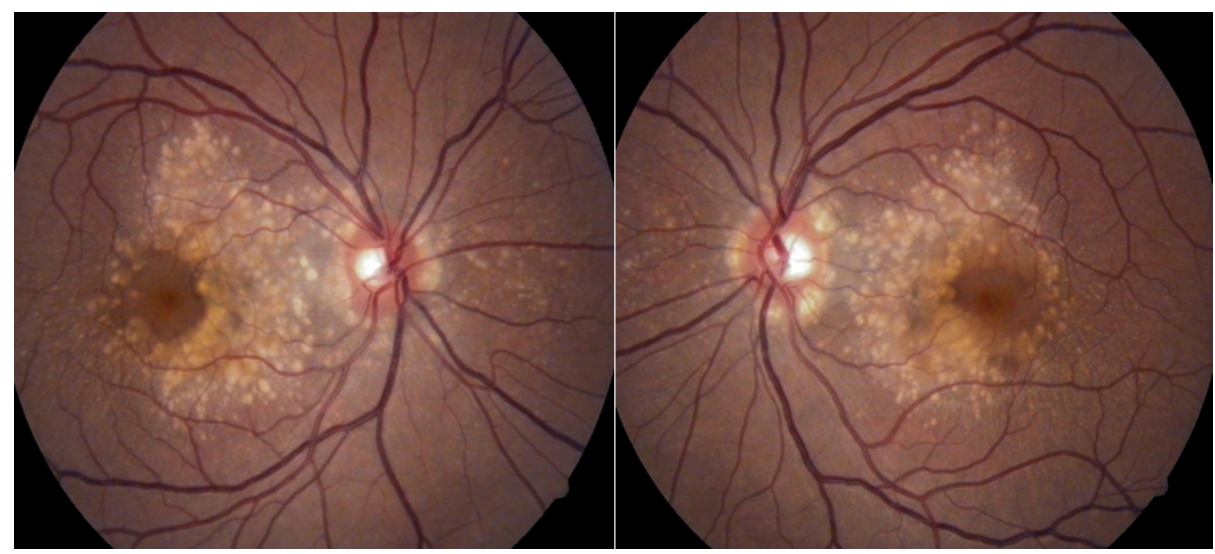

Figura 2. Foto del fondo de ojo de la paciente IV:2. En la angiografía con fluoresceína se aprecia el patrón de las drusas. 

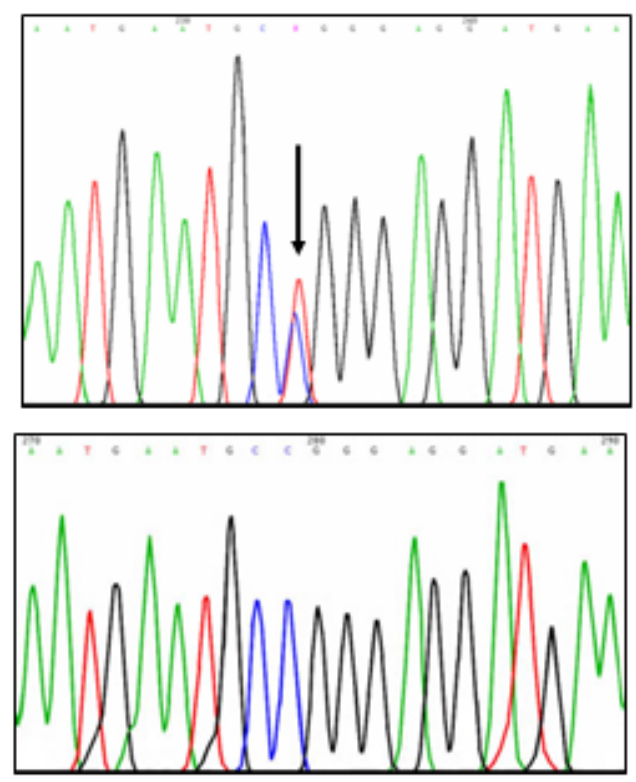

Figura 3. Electroferograma de la variante patogénica p.Arg345Trp en el gen EFEMP1.

Arriba: secuencia con variante patogénica. Abajo: secuencia normal

A la paciente III:3, afectada también con disminución de la agudeza visual, no fue posible practicarle los exámenes paraclínicos. Sin embargo, en el examen de fondo de ojo se observaron lesiones amarillentas centrales en el área macular y otras con distorsión del área de la fóvea alternando con áreas de hiperpigmentación.

Los otros dos individuos incluidos en el estudio (IV:3 y IV:4) no presentaban disminución de la agudeza visual y en el examen del fondo de ojo no se reportaron alteraciones evidentes. Cabe destacar que el hijo de la paciente IV:2 era un menor de edad asintomático, razón por la cual no se le tomó muestra para el estudio molecular. En el examen del oftalmólogo pediatra, no se reportaron alteraciones en la retina.

\section{Análisis molecular del gen EFEMP1}

En los individuos III:2, III:3 y IV:2, se detectó la variante patogénica p.Arg345Trp en el gen EFEMP1 en estado heterocigoto (figura 3). Esta variante no se halló en los individuos sanos IV:3 y IV:4.

\section{Discusión}

La correlación entre los hallazgos clínicos de las tres mujeres afectadas, los resultados del estudio molecular y el antecedente familiar de ancestros originarios del valle del Leventino en el sur de Suiza, llevó al diagnóstico de enfermedad del valle Leventina (malattia leventinese), degeneración macular que conlleva disminución de la agudeza visual a edades tempranas, caracterizada por la presencia de drusas pequeñas de disposición radial en la periferia y drusas grandes confluyentes en el centro de la mácula, también conocidas como drusas dominantes o familiares.

La enfermedad fue inicialmente descrita en 1925 por Vogt en pacientes del valle del Leventino en Suiza. Lo usual es que se inicie de forma simétrica entre la segunda y la cuarta décadas de la vida, en algunos casos, con disminución 
de la agudeza visual o metamorfopsias; no obstante, muchas otras personas pueden permanecer asintomáticas hasta edades más avanzadas.

El principal diagnóstico diferencial por fenotipo es la distrofia retiniana en panal de abejas de Doyne (3) y, aunque algunos autores han planteado que probablemente se trate de la misma enfermedad (2), otros afirman que son diferentes y que se distinguen por la variable disposición de las drusas; lo clásico en la malattia leventinese es que se sitúen en un patrón radial o radio de rueda (5). Otro diagnóstico diferencial es la degeneración macular relacionada con la edad, pero debe tenerse en cuenta que la principal diferencia está en la edad de inicio de la enfermedad, pues en la malattia leventinese generalmente se inicia entre la segunda y la cuarta décadas de la vida, en tanto que la degeneracion macular aparece en edades más avanzadas $(2,4)$.

La variante patogénica p.Arg345Trp, identificada en esta familia, es la única asociada con la enfermedad hasta el momento en diferentes poblaciones (1,58). En los estudios en pacientes de diferentes etnias, solo una vez se logró determinar esta variante en un ancestro común de origen suizo (1).

La edad de inicio de la enfermedad coincide con lo reportado por otros autores, ya que las mujeres afectadas manifestaron los primeros síntomas a los 27 y los 28 años. Como ya se dijo, los síntomas generalmente aparecen entre los 20 y los 40 años (12); sin embargo, en estas pacientes el diagnóstico clínico no se estableció a la edad de inicio de los síntomas, sino más tarde. Los hallazgos característicos incluyen drusas de inicio temprano en el polo posterior, a menudo dispuestas en una configuración radial, así como depósitos en el margen del disco óptico, drusas nasales en el disco óptico, atrofia del epitelio pigmentario de la retina en la mácula y la formación de membranas neovasculares subretinianas (13).

En la paciente III:2, los exámenes paraclínicos se practicaron de forma tardía, a pesar de que los síntomas oculares comenzaron a los 28 años, pero solo se detectaron cambios pigmentarios retinianos inespecíficos y muy avanzados que no permitieron hacer un diagnóstico; algo similar ocurrió con su hermana (paciente III:3). Por el contrario, al consultar al oftalmólogo especialista en retina y vítreo, la paciente IV:2, cuyos síntomas se iniciaron a los 27 años, fue remitida a genética con sospecha de una alteración retiniana hereditaria. En la consulta de genética médica, se detectaron los casos de los otros familiares, se identificó el patrón hereditario de la enfermedad y se encontró un ancestro originario del sur de Suiza.

Por último, fue el estudio molecular del gen EFEMP1 lo que permitió el diagnóstico clínico en esta familia. En el cuadro 1 se presentan los hallazgos de los exámenes paraclínicos de las pacientes IV:2 y III:2. Es importante resaltar que las manifestaciones clínicas son más evidentes y características de la enfermedad en etapa temprana. En la angiografía con fluoresceína de la paciente IV:2, se halló el patrón de drusas (figura 2), en tanto que, en la paciente III:2, no se evidenció una diferenciación de este patrón.

El hecho de que uno o varios individuos de una misma familia consulten muy tardíamente, o lo hagan por separado, lleva a que los servicios de oftalmología general no sospechen la condición hereditaria y los afectados no obtengan un diagnóstico específico en muchos años. Este es un llamado a los oftalmólogos generales y a los especializados en retina y vítreo a indagar más exhaustivamente sobre los antecedentes familiares y a remitir 
al genetista casos como este, en el que no se había indagado ni preguntado sobre el origen suizo de la familia.

El gen EFEMP1 codifica para la proteína fibulina-3, una glucoproteína de matriz extracelular. Aunque su función aún no se comprende por completo, hay hallazgos que la involucran en una gran cantidad de procesos fisiopatológicos (14). Los datos recopilados sugieren que una mutación en este gen puede inducir la enfermedad mediante un mecanismo de ganancia de función (1). Se ha propuesto que la acumulación de fibulina-3 mal plegada en el retículo endoplasmático de las células del epitelio pigmentario retiniano induce al estrés de dicho retículo y la activación de la reacción de proteína desplegada (unfolded protein response, UPR) (15). Esta reacción es una cascada de señalización celular adaptativa que facilita las vías de eliminación y degradación de las proteínas del retículo endoplasmático (16), proceso que podría estimular la aparición de características reconocidas de la malattia leventinese, incluida la disfunción del epitelio pigmentario retiniano, la apoptosis, la eventual atrofia y la nueva irrigación coroidea (15).

El diagnóstico molecular de las distrofias maculares relacionadas con la edad es cada vez más relevante, pues permite precisar el diagnóstico. Se han descrito distrofias maculares monogénicas que también se caracterizan principalmente por drusas del polo posterior (13). El diagnóstico molecular abre la posibilidad de un tratamiento oportuno que permita retrasar la progresión de enfermedades que amenazan la visión, como la malattia leventinese.

Además, es importante que los pediatras y los oftalmólogos con subespecialidad en pediatría estén alerta cuando haya menores de edad en familias con una enfermedad autosómica dominante, pues existe una probabilidad del $50 \%$ de heredar el gen afectado. El dilema ético implícito en la decisión de hacer la prueba molecular está entre hacer un diagnóstico precoz que puede beneficiar la calidad de vida del niño y enfrentar las consecuencias muy bien conocidas de hacer un diagnóstico molecular presintomático.

En este caso, se propuso a los padres hacer un seguimiento clínico oftalmológico permanente, de manera que, al momento de evidenciarse el más mínimo síntoma o signo de la enfermedad, se procediera a la prueba molecular a partir de una sospecha diagnóstica específica. De ahí que sea preciso alertar a los especialistas pediátricos a estar atentos ante este tipo de situaciones.

Dado que no ha habido reportes previos de esta enfermedad en una familia colombiana, este estudio brinda una herramienta muy útil para la asesoría genética y una base para futuros estudios relacionados con los diagnósticos diferenciales de la degeneración macular relacionada con la edad.

\section{Agradecimientos}

Agradecemos especialmente a la familia afectada por participar en el estudio, al Instituto de Genetica Humana de la Pontificia Universidad Javeriana, a la Fundacion Oftalmologica Nacional (FUNDONAL), a la Fundacion Derecho a la Desventaja (FUNDALDE) y al Ministerio de Ciencia, Tecnología e Innovación. 


\section{Referencias}

1. Stone EM, Lotery AJ, Munier FL, Heón E, Piguet B, Guymer RH, et al. A single EFEMP1 mutation associated with both Malattia Leventinese and Doyne honeycomb retinal dystrophy. Nat Genet. 1999;22:199-202. https://doi.org/10.1038/9722

2. Marmorstein LY, Munier FL, Arsenijevic Y, Schorderet DF, McLaughlin PJ, Chung D, et al. Aberrant accumulation of EFEMP1 underlies drusen formation in Malattia Leventinese and age-related macular degeneration. Proc Natl Acad Sci USA. 2002;99:13067-72. https://doi.org/10.1073/pnas.202491599

3. Souied EH, Leveziel N, Letien V, Darmon J, Coscas G, Soubrane G. Optical coherent tomography features of Malattia Leventinese. Am J Ophthalmol. 2006;141:404-7. https://doi.org/10.1016/j.ajo.2005.09.001

4. Johnson LV, Anderson DH. Age-related macular degeneration and the extracellular matrix. N Engl J Med. 2004;351:320-2. https://doi.org/10.1056/NEJMp048131

5. Pager CK, Sarin LK, Federman JL, Eagle R, Hageman G, Rosenow J, et al. Malattia Leventinese presenting with subretinal neovascular membrane and hemorrhage. Am J Ophthalmol. 2001;131:517-8. https://doi.org/10.1016/s0002-9394(00)00821-7

6. Matsumoto M, Traboulsi El. Dominant radial drusen and Arg345Trp EFEMP1 mutation. Am J Ophthalmol. 2001;131:810-2. https://doi.org/10.1016/s0002-9394(00)00926-0

7. Guymer R. The genetics of age-related macular degeneration. Clin Exp Optom. 2001;84:1829. https://doi.org/10.1111/j.1444-0938.2001.tb05023.x

8. Zhan T, Xie X, Cao G, Jiang H, Wu S, Su Z, et al. Malattia Leventinese/Doyne honeycomb retinal dystrophy in a Chinese family with mutation of the EFEMP1 gene. Retina. 2014;34:2462-71. https://doi.org/10.1097//AE.0000000000000259

9. Khan KN, Mahroo OA, Khan RS, Mohamed MD, McKibbin M, Bird A, et al. Differentiating drusen: Drusen and drusen-like appearances associated with ageing, age-related macular degeneration, inherited eye disease and other pathological processes. Prog Retin Eye Res. 2016;53:70-106. https://doi.org/10.1016/j.preteyeres.2016.04.008

10. Ardeljan D, Chan CC. Aging is not a disease: Distinguishing age-related macular degeneration from aging. Prog Retin Eye Res. 2013;37:68-89. https://doi.org/10.1016/j.preteyeres.2013.07.003

11. Guymer RH. Splitting the lumps: The importance of phenotyping drusen. Ophthalmol. 2018;125:6-7. https://doi.org/10.1016/j.ophtha.2017.09.007

12. Narendran N, Guyner RH, Cair M, Baird PN. Analysis of the EFEMP1 gene in individuals and families with early onset drusen. Eye (Lond.). 2005;19:11-5. https://doi.org/10.1038/sj.eye.6701435

13. Michaelides M, Jenkins SA, Brantley MA, Andrews RM, Waseem N, Luong V, et al. Maculopathy due to the R345W substitution in fibulin-3: Distinct clinical features, disease variability, and extent of retinal dysfunction. Invest Ophthalmol Vis Sci. 2006;47:3085-97. https://doi.org//10.1167/iovs.05-1600

14. Livingstone I, Uversky VN, Furniss D, Wiberg A. The pathophysiological significance of fibulin-3. Biomolecules. 2020;10:1294. https://doi.org//10.3390/biom10091294

15. Roybal CN, Marmorstein LY, Vander Jagt DL, Abcouwer SF. Aberrant accumulation of fibulin-3 in the endoplasmic reticulum leads to activation of the unfolded protein response and VEGF expression. Investig Ophthalmol Vis Sci. 2005;46:3973-9. https://doi.org//10.1167/iovs.05-0070

16. Ron $D$, Walter $P$. Signal integration in the endoplasmic reticulum unfolded protein response. Nat Rev Mol Cell Biol. 2007;8:519-29. https://doi.org//10.1038/nrm2199 